\title{
Guidelines of care for clinical laser treatments: why are they useful?
}

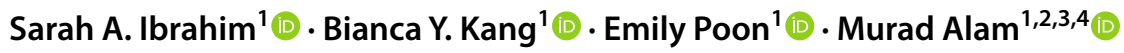 \\ Received: 17 August 2021 / Accepted: 15 November 2021 / Published online: 30 November 2021 \\ (c) The Author(s), under exclusive licence to Springer-Verlag London Ltd., part of Springer Nature 2021
}

\section{Dear Editor:}

Clinical laser treatments are now so routine that it is only to be expected that some degree of standardization would emerge. A particular type of standard increasingly evident in clinical laser care is the clinical practice guideline. [1-3] Clinical practice guidelines are based on literature and expert opinion.[4-8] The purpose of such guidelines is to identify clinical questions that are important to patients and practitioners and then seek to provide relevant recommendations. These recommendations, which often pertain to the treatment of a particular disease or condition, help to guide physicians in developing treatment plans for individual patients while considering the amount of evidence supporting each clinical decision.

While guidelines may be relatively concise, the developmental process that births them can be long and arduous. Indeed, there are guidelines for guideline development, notably Reporting Items for practice Guidelines in HealThcare (RIGHT) and Appraisal of Guidelines, Research and Evaluation (AGREE II). [9, 10] The first step is to constitute a group of experts, most of whom should not have relevant conflicts of interest. Before research can get underway, the guidelines group must identify important clinical questions that bear investigation. Solicitation of such questions may lead to the creation of a long list, which may be culled. Questions that remain are then formally investigated via a comprehensive literature search and, often, meta-analysis. Evidence is

Murad Alam

m-alam@northwestern.edu

1 Department of Dermatology, Feinberg School of Medicine, Northwestern University, Chicago, IL, USA

2 Department of Otolaryngology, Feinberg School of Medicine, Northwestern University, Chicago, IL, USA

3 Department of Surgery, Feinberg School of Medicine, Northwestern University, Chicago, IL, USA

4 Department of Medical Social Sciences, Feinberg School of Medicine, Northwestern University, Chicago, IL, USA reviewed by groups of experts, who consider it in the context of their experience and other unpublished information. One or more recommendations may be offered pertaining to each question posed, with some such being weak or strong, depending on the underlying evidence. In some cases, the sum of evidence and expert opinion may not permit even a weak recommendation. Even when recommendations are provided, caveats and exceptions are typically discussed, as is the need to consider patient-specific factors. Blind adherence to clinical recommendations is neither expected nor appropriate.

For clinical laser treatment, many sets of guidelines are available. Some are from professional societies, some from collaborations among such societies, and some from groups specially constituted to create guidelines. In response to the COVID-19 crisis, living guidelines have also been developed in record time. As the clinical laser community is of modest size, guidelines may even be international in scope when addressing very specific issues or treatment types.

Though available, guidelines may not necessarily be routinely implemented into clinical practice. This may be particularly true in the case of laser and energy-based treatments, since patients often pay out of pocket for procedures with these devices. As a result, providers are not required to adhere to standards set forth by government or private insurance. Still, guidelines can help to facilitate care and foster the patient-physician relationship. By providing a concise summary of both expert opinion and literature review, guidelines are an efficient source of information. This information - which often includes specific recommendations for treatment protocols and patient selection, as well as details about expected outcomes, and management and prevention of adverse events-may help to guide the physician in clinical decision-making and also form the basis of conversation with patients.

In summary, the purpose of guidelines is not to appropriate physician autonomy but rather to help patients and physicians benefit from accumulated wisdom. On the one hand, guidelines are derived from high level evidence carefully sifted by experts. However, much remains unknown, 
especially in rapidly advancing fields like laser medicine and surgery, and physicians must continue to exercise good judgment.[11] Even when guidance is available, it may not apply to patients with unusual presentations. It remains the physician's purview to incorporate guideline recommendations when and if they are appropriate.

Data availability Not applicable.

Code availability Not applicable.

\section{Declarations}

Ethics approval Not applicable.

Consent to participate Not applicable.

Consent for publication Not applicable.

Conflict of interest The authors declare no competing interests.

\section{References}

1. Waldman A, Bolotin D, Arndt KA et al (2017) ASDS Guidelines Task Force: Consensus Recommendations Regarding the Safety of Lasers, Dermabrasion, Chemical Peels, Energy Devices, and Skin Surgery During and After Isotretinoin Use. Dermatol Surg Off Publ Am Soc Dermatol Surg Al 43(10):1249-1262. https:// doi.org/10.1097/DSS.0000000000001166

2. Seago M, Shumaker PR, Spring LK et al (2020) Laser Treatment of Traumatic Scars and Contractures: 2020 International Consensus Recommendations. Lasers Surg Med 52(2):96-116. https:// doi.org/10.1002/1sm.23201
3. Alam M, Dover JS, Arndt KA (2007) Use of cutaneous lasers and light sources: appropriate training and delegation. Skin Ther Lett 12(5):5-9

4. Alam M, Harikumar V, Kang BY, et al (2020) Development of international clinical practice guidelines: benefits, limitations, and alternative forms of international collaboration. Arch Dermatol Res. https://doi.org/10.1007/s00403-020-02166-y

5. Harikumar V, Worley B, Ibrahim SA, et al (2020) Broad versus narrow clinical practice guidelines: avoiding rules for the high risk 1. Arch Dermatol Res. https://doi.org/10.1007/ s00403-020-02160-4

6. Worley B, Kyllo R, Harikumar V, et al. Comparative utility of appropriate use criteria versus clinical practice guidelines. Arch Dermatol Res. https://doi.org/10.1007/s00403-020-02161-3

7. Sobanko JF, Harikumar V, Kang BY, et al (2020) Multisociety and multispecialty clinical practice guidelines. Arch Dermatol Res. https://doi.org/10.1007/s00403-020-02162-2

8. Owen JL, Kibbi N, Worley B et al (2019) Sebaceous carcinoma: evidence-based clinical practice guidelines. Lancet Oncol 20(12):e699-e714. https://doi.org/10.1016/S1470-2045(19) 30673-4

9. Hoffmann-Eßer W, Siering U, Neugebauer EAM, Brockhaus AC, Lampert U, Eikermann M (2017) Guideline appraisal with AGREE II: Systematic review of the current evidence on how users handle the 2 overall assessments. PLoS ONE 12(3):e0174831. https://doi.org/10.1371/journal.pone.0174831

10. Wayant C, Cooper C, Turner D, Vassar M (2019) Evaluation of the NCCN guidelines using the RIGHT Statement and AGREEII instrument: a cross-sectional review. BMJ Evid-Based Med 24(6):219-226. https://doi.org/10.1136/bmjebm-2018-111153

11. Fricton R, Poon E, Weil A, Alam M (2020) Office practices that may improve the experience of the cosmetic dermatology patient. Arch Dermatol Res. https://doi.org/10.1007/s00403-020-02154-2

Publisher's Note Springer Nature remains neutral with regard to jurisdictional claims in published maps and institutional affiliations. 